\title{
A LOW-TEMPERATURE THERMOPNEUMATIC GAS BUBBLE VALVE
}

\author{
Wouter van der Wijngaart, Dipankar Chugh, Elisabeth Man, Jessica Melin* and Göran Stemme
}

\author{
Microsystem Technology, School of Electrical Engineering, Royal Institute of Technology, Stockholm, \\ SWEDEN
}

URL: http://www.s3.kth.se/mst, email:wouter@s3.kth.se

\begin{abstract}
We introduce a novel type of actuation for microvalves, in which a trapped air bubble functions as the thermally controlled volume displacing element. The pressure of the trapped air pocket is controlled thermally using a resistive heater. The volume displacement is determined by the liquid surface tension and the valve geometry. A fully functional demonstrator device was successfully fabricated and tested. The absence of moving mechanical parts, the electrical control of the valve, and the limited required actuation temperature make the actuator suitable for control of LSI fluidic networking in biotechnical applications.
\end{abstract}

\section{INTRODUCTION}

Microvalves form one of the fundamental components of microfluidic based devices. Their functionality is analogous to that of transistors in electronics.

Gas bubbles have been used as active elements in reversible volume displacing microfluidic actuators. They are formed and controlled by a liquid-gas phase change, either through boiling or electrolysis [1]. The current work introduces a new type of gas bubble actuator, in which the active gas bubble consists of gas that is trapped during device priming. The volume displacing bubble actuation utilises thermal expansion and phase equilibrium shift (i.e. vapour saturation pressure and air solubility) with temperature for actuation, allowing device control at temperatures well below the boiling temperature of the liquid.

The microfluidic actuation approach introduced in this paper features a number of benefits in comparison with previous work. It allows downscaling microvalve elements to a size of $10 \mu \mathrm{m}$ or below. The valves are controlled electrically, thus providing the potential for a fully portable system which is much more difficult with pneumatically driven systems [2]. The novel approach is thus suited for large scale integration (LSI) of active fluidic control elements. Moreover, the valves consume low power, because no boiling heat is required and because there is no active

*Jessica Melin's current address is Stanford University, Bioengineering, Quake Lab, 318 Campus Drive, Clark Center E300, Stanford, CA 94305-5432, USA. element (membrane, diaphragm) with inherent mechanical stiffness to be accounted for. The moderate temperatures required to operate the valves make them suitable for biological assays, in particular samples prone to thermal denaturation. The actuation principle here does not rely on electrical fields or field gradients in the liquid, such as electro-osmotically controlled flow, thus making the combination of such actuation schemes uncomplicated.

\section{DEVICE DESCRIPTION AND OPERATION PRINCIPLE}

Fig. 1 shows a schematic of a microvalve using the novel actuator. It consists of a liquid microchannel, with an inlet and outlet. An actuation window connects the liquid channel with an actuation chamber. The resistive heater in the actuation chamber is used for thermopneumatic actuation.

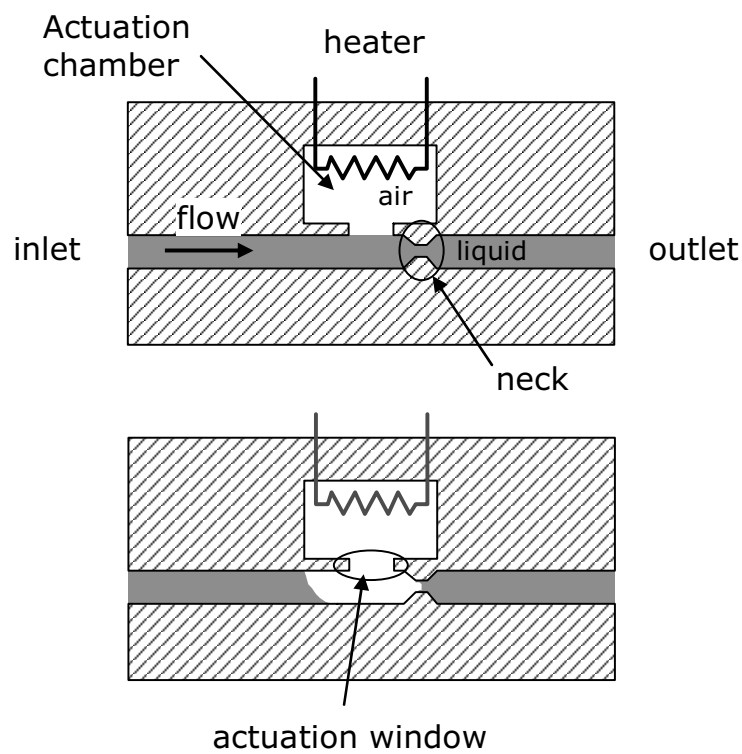

Fig. 1. Conceptual schematic of an open (top) and actuated closed (bottom) valve.

During liquid priming of the channel, a combination of surface wettability and geometry control ensures that the liquid-air-solid triple-phase line remains in the actuation window between the liquid channel and the actuation chamber, i.e. the liquid will not enter the actuation chamber, thus trapping an air pocket in the actuation chamber. The valve is open in the normal mode of operation. Closing the valve is achieved by increasing the temperature of the gas 
volume in the actuation chamber, which increases the pressure of the gas. The pressure increase introduces a gas bubble into the liquid channel, blocking the flow. Liquid flow in the channel is once again established when the gas pressure is reduced by decreasing the temperature in the actuation chamber. The microchannel downstream of the actuation window is necked such that large capillary forces hold the bubble at the actuation window and prevent it from entering the downstream system [3].

The gas pocket expansion depends strongly on the time the valve is in closed state. For a short closed valve state, there will be limited mass transfer between the liquid in the channel and the gas in the actuation chamber. Pure thermal expansion of the gas will determine the pressure in the actuation chamber. For a long closing of the valve, the system will strive to its thermodynamic equilibrium, and the pressure-temperature characteristics are determined by the saturation vapour pressure and the equilibrium gas solubility instead.

\section{DEMONSTRATOR FABRICATION}

We fabricated and successfully tested a number of mesoscale microvalve demonstrators, see Fig. 4. The specific device layout and fabrication method and materials were chosen to allow quick, uncomplicated and flexible fabrication and valve interfacing.

The demonstrator valves are 3-layer structures, consisting of 1) a glass substrate, 2) a thin PDMS sheet containing the liquid channel and actuation window, and 3) a thick PDMS block containing fluidic interconnect channels for the valve inlet and outlet, and the actuation chamber. The liquid channel in the PDMS sheet contains square pillar structures for mechanical support during fabrication. The channel width was 100 or $200 \mu \mathrm{m}$ and the depth $50 \mu \mathrm{m}$.

The thin PDMS sheet fabrication, illustrated in Fig. 2, involves the casting of PDMS on a $\mathrm{CF}_{\mathrm{x}}$-coated silicon mold in a process adapted from Hsu et al. [4]. The silicon master was fabricated using two subsequent DRIE steps and was coated with $\mathrm{CF}_{\mathrm{x}}$ polymer using plasma deposition to ease the release of the PDMS sheet after molding. PDMS prepolymer (SYLGARD 184 silicone elastomer Kit, Dow Corning) was mixed with the curing agent (10:1 weight ratio) and degassed for $30 \mathrm{~min}$ to remove the air bubbles. The mixture is dispensed on top of the silicon master and a fluorocarbon polymer coated polyestersheet (Scotchpak ${ }^{\mathrm{TM}} 1020$ release liner, 3M) is carefully rolled over the PDMS avoiding air bubbles. All excess PDMS is squeezed out by thumb since it is important that there is no material left on the top of the silicon mold. The PDMS is finally evened out by pressing a thick piece of glass onto the top surface. Thereafter the PDMS sheet is cured at $65^{\circ} \mathrm{C}$ for 4 hours. After curing, the PDMS adheres reversibly to the PE sheet and is removed from the master along with the PE sheet.

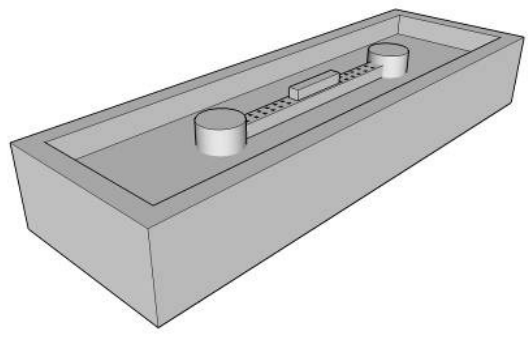

a. The silicon master is coated with a $C F_{x}$ polymer.

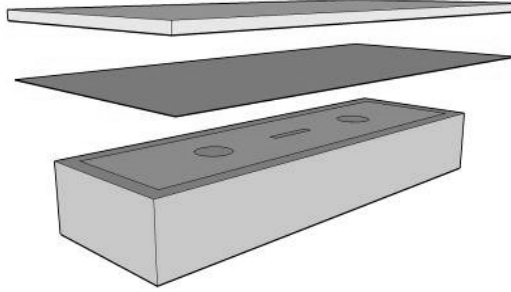

b. Excess PDMS is squeezed out; the mold is covered with Scotchpak ${ }^{\mathrm{TM}}$ release liner; and pressed with a glass piece.

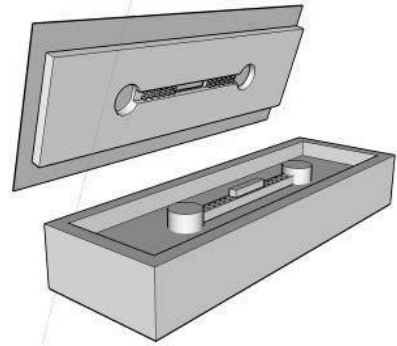

c. After curing, the PDMS sheet can be easily removed from the mold and from the master.

d.

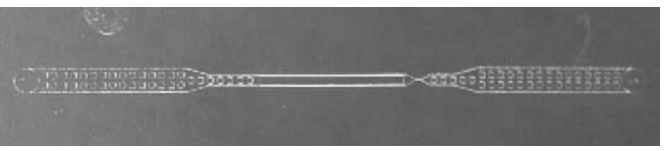

Fig. 2. a to c: Step by step schematic of the fabrication of the thin PDMS sheet. d: microscope pictures of PDMS sheets with $200 \times 3000 \mathrm{\mu m}^{2}$ actuation window.

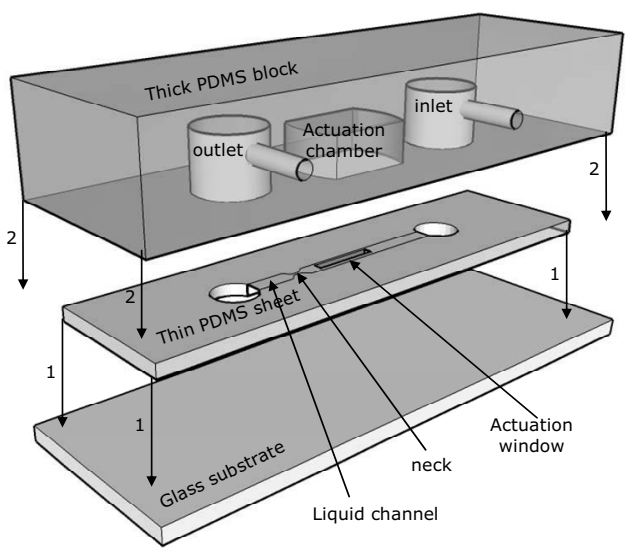

Fig.3. Exploded schematic of the device assembly. The subsequent bonding steps are numbered. 
The thick PDMS block is cast in a mold. The mold inserts are removed after the PDMS is cured. A $100 \mathrm{Ohm} \mathrm{Pt}$ resistance temperature detector (Heraeus M-FK 222) with size $0.8 * 2.2 * 2.3 \mathrm{~mm}^{3}$ is used simultaneously as heater and temperature sensor and placed inside the actuation chamber. Its electrical leads are punched through the PDMS for contacting. Fig. 3 illustrates the assembly of the valve. First, the PDMS sheet is peeled from the PE sheet and placed on the glass substrate, creating a reversible bond. Thereafter the thick PDMS block is bonded on top of the PDMS sheet by applying gentle pressure. Fig. 4 shows photographs of the complete valve.

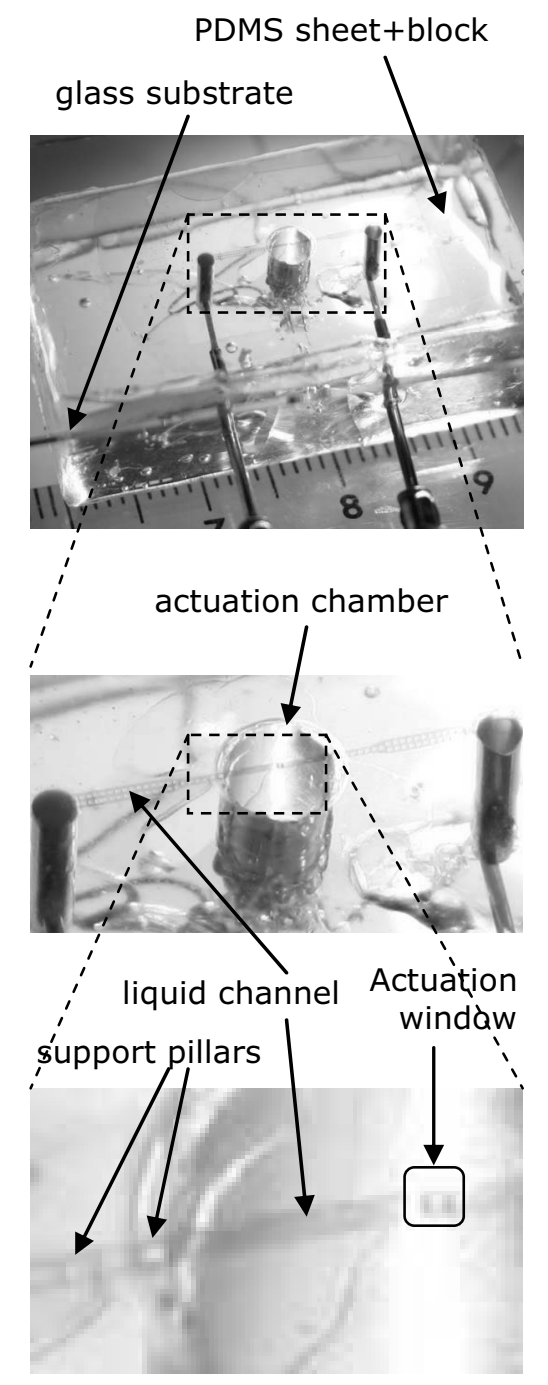

Fig. 4. Photograph of a demonstrator valve (left) with a close-up (middle and right). The liquid is dyed red for visibility. One cannot distinguish the thick PDMS block and thin PDMS sheet in the photographs.

\section{EXPERIMENTAL}

A measurement setup was built as illustrated in Fig. 5. When the syringe pump is in the off-state, the inlet and outlet pressure can be applied hydrostatically. Alternatively, a flow can be established through the device with the syringe pump, while the inlet and outlet pressure are measured hydrostatically. The heater is coupled to a voltage source, and voltage and current were continuously monitored. In the below valve characterisation, we used DI water to which we added red dye for visualisation, as well as detergent for easy priming of the structure. The surface tension of the liquid thus has an estimated value $\gamma=25 \mathrm{mN} / \mathrm{m}$. In a first test, the valve opening and closing was successfully evaluated (Fig. $6)$.

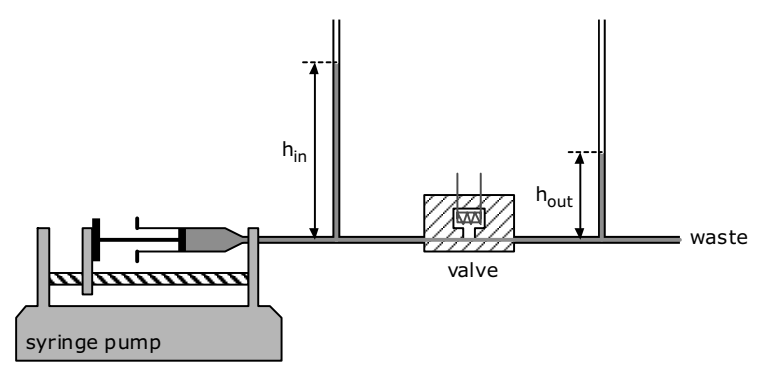

Fig. 5. Schematic of the measurement setup.

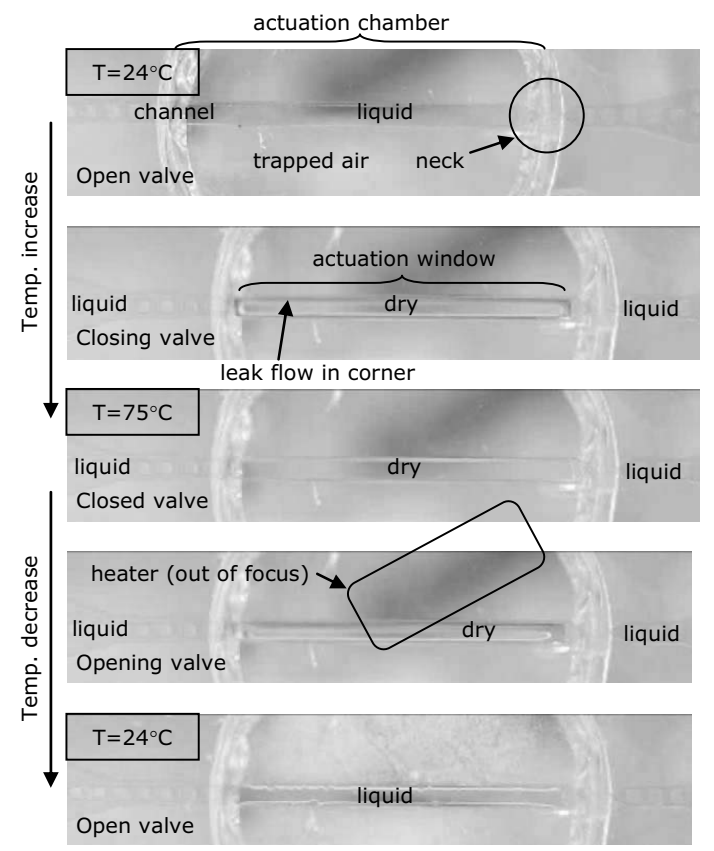

Fig. 6. Video frame sequence of the closing and opening of a valve (top to bottom).

In a next set of measurements, the power required to close the valve against a specific liquid pressure was determined (Fig. 7). For each subsequent measurement, both the inlet and outlet pressure were increased to an equal value, with no flow through the valve. Thereafter, the power through the heater was gradually increased until the valve was closed. Based on these measurements, the actuation pressure generated in the actuation chamber can be estimated by adding an estimated phase interface pressure $\operatorname{drop}_{g}-P_{l} \cong \frac{4 \gamma}{w}=1 \mathrm{kPa}$ to the applied liquid pressure, $w$ being the window width. One can observe that in this particular valve, a temperature rise of $10^{\circ} \mathrm{C}$ is sufficient to close the valve against pressures of a few $\mathrm{kPa}$. 

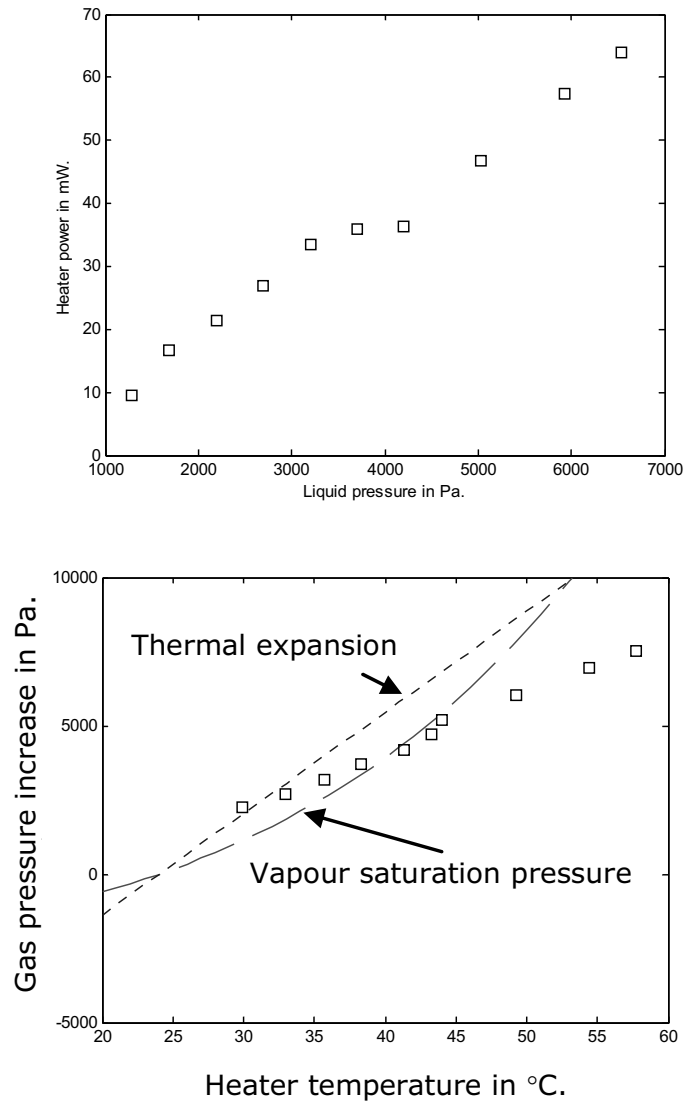

Fig. 7. Top: applied heating power to close the valve against a set liquid pressure; Bottom: calculated gas pressure and calculated heater temperature for the same measurement data. The blue dotted line indicates the theoretical expected gas pressure for pure thermal expansion; the red dashed line indicates the theoretical stationary gas pressure caused by vapour saturation pressure.

\section{DISCUSSION}

Three operation limitations were observed: downstream bubble introduction, flooding, and parasitic offset pressure due to non-equilibrium priming conditions. Flooding, schematically described in Fig. 8, occurs when the liquid-gas pressure drop $\mathrm{P}_{1}-\mathrm{P}_{\mathrm{g}}$ forces the solid-liquid-gas triple phase line to detach from the actuation window and enter the actuation chamber. For hydrophobic actuation chamber surfaces, flooding occurs when $\mathrm{P}_{1}-\mathrm{P}_{\mathrm{g}}$ reaches a value somewhere between $\frac{2 \gamma}{w}$ and $\frac{4 \gamma}{w}$, depending on the exact actuation window geometry.

Downstream bubble introduction refers to when the liquid in the channel splits off a gas bubble from the actuation chamber and drags it along in the channel. The latter occurs when the liquid pressure drop $\mathrm{P}_{\text {in }}-\mathrm{P}_{\text {out }}$ over the actuation window region of the valve is larger than the capillary pressure in the liquid channel neck. Thus, the neck width must be made small enough to withstand the liquid pressure range controlled by the valve. During our experiments, bubble introduction was observed regularly for valves without channel necking, but never for valves with neck.
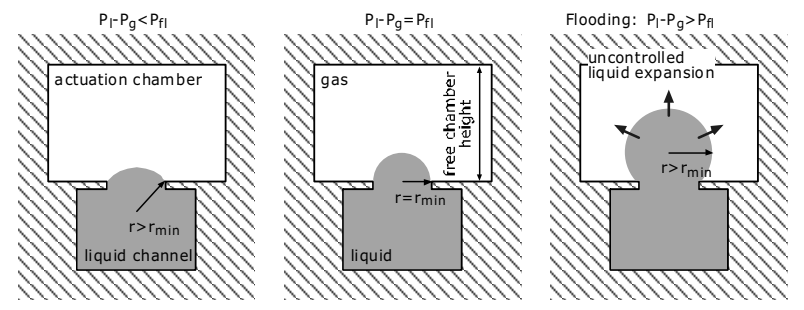

Fig. 8. Left to right: schematic of the liquid-gas interface shape during flooding for increasing values of $P_{l}-P_{g}$.

Parasitic offset pressure $P_{\text {off }}$ refers to changes in the device behaviour caused by specific atmospheric conditions at the time of liquid priming. When the atmospheric humidity is below saturation during priming, the air trapped in the actuation chamber will spontaneously start absorbing liquid from the actuation window. The chamber pressure $\mathrm{P}_{\mathrm{g}}$ will thus increase to a level above the atmospheric pressure with a specific offset, depending on the atmospheric humidity. This effect causes a shift in the valve characteristics: it decreases the required power to close the valve, and it increases the valve resistance against flooding. However, care must be taken that the shift in characteristics does not lead to the valve closing at temperatures below room temperatures (which would render the valve unusable).

Under the experimental conditions in our lab, we measured a typical offset pressure value $P_{\text {off }}=400 \mathrm{~Pa}$, and a flooding pressure $P_{f l}=2 \mathrm{kPa}$ for a valve with a $200 \mu \mathrm{m}$ square actuation window.

\section{CONCLUSIONS}

We introduce a novel type of microvalve, in which a trapped air bubble functions as the thermally controlled volume displacing element. Devices were fabricated and successfully tested. The temperature change required to switch the valve state is typically a few tens of degrees. Three fundamental valve operation limitations were observed. The microvalve principle is suited for further miniaturisation and a viable candidate for control of LSI fluidic networking in biotechnical applications.

\section{REFERENCES}

[1] Meng et al., "A comparative study of electrolysis and boiling for bubble driven microactuators", Proceedings IEEE Transducers '05, Seoul, Korea, 2005, 1263-1266.

[2] Thorsen et al., "Microfluidic large-scale integration", Science 298 (2002), 580-584.

[3] Melin et al., "Behaviour and design considerations for continuous flow closed-open-closed liquid microchannels", Lab on a Chip 5 (2005), 682-686.

[4] C-H. Hsu et al., "Microcanals for micropipette access to single cells in microfluidic environments", Lab on a Chip 4 (2004), 420-424. 